\title{
Systematic Space-Time Trellis Code Design for an Arbitrary Number of Transmit Antennas
}

\author{
Zoltan Safar and K. J. Fiay Liu \\ Department of Electrical and Computer Engineering \\ University of Maryland, College Park, MD 20742
}

\begin{abstract}
The potential for capacity increase in multiantenna wireless communication systems has drawn considerable attention to space-time codes. In this work, we propose a systematic code construction method that jointly considers diversity advantage and coding advantage for an arbitrary number of transmit antennas and any memoryless constellation. Due to the special structure of the channel symbol difference matrix, the code construction problem is reduced to a combinatorial optimization problem and a computationally efficient suboptimal solution is proposed. The simulations show that our design procedure results in codes that outperform the ones constructed by previously existing methods. In certain cases, as much as 2-2.5 dB coding gain can be observed.
\end{abstract}

Keywonds - space-time codes, trellis codes, transmit diversity, wireless communication

\section{INTRODUCTION}

The design of mobile communication systems that offer reliable transmission at high data rates is a challenging task. The transmitted signal undergoes multipath fading and interference from other users. Moreover, there are severe limitations on the available resources such as bandwidth and battery power. Therefore, future wireless systems are likely to employ diversity techniques to increase the data rate of the communication links.

Information-theoretic works [1], [2] have shown that the capacity of flat fading channels is substantially increased when using multiple transmit and/or receive antennas. If the path gains between the transmit and receive antennas are assumed to be known at the receiver side, the channel capacity is approximately proportional to the minimum of the number of transmit and receive antennas.

Space-time (ST) trellis codes have been proposed as a means to exploit the potential for capacity increase in multi-antenna systems. The performance criteria were derived in [3] and [4], characterizing the ST codes with two quantities: the diversity advantage, which describes the asymptotic error rate decrease as a function of the signai to noise ratio (SNR), and the coding advantage, which determines the vertical shift of the error performance curve. In [4], the authors proposed design rules for two transmit antennas to achieve maximum diversity advantage. They also derived a lower bound on the complexity of the encoder and the decoder for the desired diversity advantage and data throughput. This lower bound states that in order to achieve a diversity advantage of $K$ and transmit one $B$-ary source symbol per state transition, the encoder and the decoder must have at least $N_{\min }=B^{K-1}$ states.

The repetition coded delay diversity scheme described in
[5] was the first systematic design rule for arbitrary number of transmit antennas. Using this method, ST codes achieving full diversity advantage can be designed for any memoryless constellation.

In [6], the design problem was transformed into the binary domain. The authors proposed design methods for an arbitrary number of transmit antennas, but only for BPSK and QPSK constellations. Moreover, the code construction methods in [5] and [6] for full diversity advantage uniquely determine the ST codes.

The design of ST codes that achieve full spatial diversity for any number of transmit antennas and any constellation was also considered in [7]. An important feature of this code construction method is that it does not specify the ST codes completely, leaving room for additional optimization for coding advantage. However, the authors did not present a systematic design method to take advantage of the additional freedom.

In this paper, we develop a code construction procedure that jointly considers diversity advantage and coding advantage for an arbitrary number of transmit antennas and any memoryless constellation. We describe a method to maximize the coding advantage based on the design rules of [7] for diversity advantage. The code construction problem is formulated as a combinatorial optimization problem, and a computationally efficient suboptimal solution is proposed.

The paper is organized as follows. Section II will introduce the mathematical model of the communication system. The relevant results of previous works will be briefly restated in Section III. The code construction method will be developed in Section IV. Section V will describe a specific ST code design example. The simulation results will be provicled in Section VI, and some conclusions will be drawn in Section VII.

\section{SYSTEM MODEL}

Consider a wireless communications system with $K$ transmit and $L$ receive antennas. The input bit stream is divided! into $b$ bit long blocks, forming $B$-ary $\left(B=2^{b}\right)$ source symbols. The ST encoder works as a finite state machine with $N$ states: it takes the current source symbol, $b_{t}\left(b_{t} \in\{0,1, \ldots, B-1\}\right)$ at discrete time $t$, and governed by this input and the current state, $S_{t}\left(S_{t} \in\{0,1, \ldots, N-1\}\right)$, it moves to the next state, $S_{t+1}$. During this state transition, the encoder outputs $K B$-ary channel symbol indices. We denote by $i^{k}\left(S_{t}, b_{t}\right)$ the channel symbol index for an- 
tenna $k, k=0,1, \ldots, K-1$, generated during the state transition from $S_{t}$ when the current source symbol is $b_{t}$. We will also use the channel symbol index vector, defined as:

$$
\mathbf{i}\left(S_{t}, b_{t}\right)=\left[i^{0}\left(S_{t}, b_{t}\right), i^{1}\left(S_{t}, b_{t}\right), \ldots, i^{K-1}\left(S_{t}, b_{t}\right)\right]^{T} .
$$

These channel symbol indices are mapped onto channel symbols (or constellation points) by the modulators and transmitted through the transmit antennas. In the sequel, $c(i)$ will represent the constellation point corresponding to channel symbol index $i$. (For example, in case of $B$-ary PSK, $c(i)=\exp (j 2 \pi i / B)$, where $j=\sqrt{-1}$.) All the constellations are assumed to be normalized so that the average energy of the constellation is unity (if the channel symbols are equally likely). $c\left(i^{k}\left(S_{t}, b_{t}\right)\right)$ will denote the constellation point output by antenna $k$ when the current state is $S_{t}$ and the current input is $b_{t}$. In vector notation:

$\mathbf{c}\left(S_{t}, b_{t}\right)=\left[c\left(i^{0}\left(S_{t}, b_{t}\right)\right), c\left(i^{1}\left(S_{t}, b_{t}\right)\right), \ldots, c\left(i^{K-1}\left(S_{t}, b_{t}\right)\right)\right]^{T}$.

The transmission medium is assumed to be flat (frequency nonselective), quasi-static Rayleigh fading channel. The quasi-static property means that the channel remains constant over a certain time, called the frame period, and changes independently from one frame to the other. The path gains are assumed to be known by the receiver. They are modeled as independent, complex, zero mean, circularly symmetric Gaussian random variables with unit variance.

At the receiver side, the received signals at each receive antenna are demodulated, and the ST decoder produces the decoded bit stream. The receiver noise is modeled as independent, complex, zero mean, circularly symmetric Gaussian random variables.

\section{DESIGN FOR DIVERSITY ADVANTAGE}

Assume that the previously described transmitter sends $T(T>K) B$-ary source symbols to the receiver. The ST encoder, while encoding the source symbol sequence $\left\{b_{t}\right\}$, goes through the sequence of states $\left\{S_{t}\right\}$, and produces $\mathbf{C}$, the $K$ by $T$ channel symbol matrix:

$$
\mathbf{C}=\left[\mathbf{c}\left(S_{0}, b_{0}\right), \mathbf{c}\left(S_{1}, b_{1}\right), \ldots, \mathbf{c}\left(S_{T-1}, b_{T-1}\right)\right] .
$$

The decoder, due to decoding errors, goes through a different sequence of states, $\left\{S_{t}^{\prime}\right\}$, producing the erroneously decoded source symbol sequence $\left\{b_{t}^{\prime}\right\}$ and the $K$ by $T$ channel symbol matrix $\mathbf{C}^{\prime}$ :

$$
\mathbf{C}^{\prime}=\left[\mathbf{c}\left(S_{0}^{\prime}, b_{0}^{\prime}\right), \mathbf{c}\left(S_{1}^{\prime}, b_{1}^{\prime}\right), \ldots, \mathbf{c}\left(S_{T-1}^{\prime}, b_{T-1}^{\prime}\right)\right] .
$$

Define $\mathbf{D}$, the channel symbol difference matrix, as $\mathbf{D}=\mathbf{C}-\mathbf{C}^{\prime}$, and a $K$ by $K$ matrix $\mathbf{A}$ as $\mathbf{A}=\mathbf{D D}^{H}$. The design criteria [4] were derived to minimize the probability that the decoder erroneously decodes $\mathbf{C}^{\prime}$ if $\mathbf{C}$ was sent:

1. Design for full spatial diversity (rank criterion): The matrix $\mathbf{A}$ (or equivalently, the matrix $\mathbf{D}$ ) must be of full rank for any distinct $\mathbf{C}$ and $\mathbf{C}^{\prime}$ matrices.

2. Design for coding advantage (determinant criterion): The minimum determinant of $\mathbf{A}$ taken over all distinct $\mathbf{C}$ and $\mathbf{C}^{\prime}$ matrices must be as large as possible. If the minimum determinant is $\gamma$, then a coding advantage of $\sqrt[k]{\gamma}$ has been achieved.

Without loss of generality, we can assume that the first decoding error occurs at $S_{0}$, so the correct and decoded paths diverge at this point (i.e. $S_{0}=S_{0}^{\prime}$ and $b_{0} \neq b_{0}^{\prime}$ ). The code construction method proposed in [7] ensured full spatial diversity by making the matrix

$$
\begin{aligned}
\mathbf{D}_{1}=\left[\mathbf{c}\left(S_{0}, b_{0}\right)-\mathbf{c}\left(S_{0}^{\prime}, b_{0}^{\prime}\right), \ldots\right. \\
\left.\quad \ldots, \mathbf{c}\left(S_{K-1}, b_{K-1}\right)-\mathbf{c}\left(S_{K-1}^{\prime}, b_{K-1}^{\prime}\right)\right],
\end{aligned}
$$

which consists of the first $K$ columns of the code difference matrix D, upper triangular for any possible correct and erroneous paths through the trellis. The design method is based on the following definitions:

Definition 1: A level $t$ group is a collection of all destination states that can be reached at state transition $t$ from a given $S_{0}$ starting state through all possible $b_{0}, b_{1}, \ldots, b_{t-1}$ input sequences.

Definition 2: A subgroup of a level $t$ group is a collection of all destination states that can be reached at state transition $t$ from a given $S_{0}$ starting state and a given $b_{0}$ starting branch through all possible $b_{1}, b_{2}, \ldots, b_{t-1}$ input sequences.

It can be shown that the above definitions have the following properties for $t=1,2, \ldots, K-1$ :

1. Any level $t$ group starts at state $m$ such that $m \bmod$ $B^{t}=0$ and consists of $B^{t}$ consecutive states.

2. Any subgroup of a level $t$ group starts at state $m$ such that $m \bmod B^{t-1}=0$ and consists of $B^{t-1}$ consecutive states.

3. Every level $t$ group consists of $B$ disjoint subgroups.

4. $S_{t}$ and $S_{t}^{\prime}$ belong to the same level $t$ group.

5. For some $l \neq m\left(l, m \in\{0,1, \ldots B-1\}, S_{t}\right.$ belongs to the $l$ th and $S_{t}^{\prime}$ belongs to the $m$ th subgroup of the same level $t$ group.

The code construction rules are [7]:

(1a) The 0th indices of the channel symbol index vectors at the same state must be different.

(1b) The remaining indices of the channel symbol index vectors at the same state must be the same.

(2a) For $t=1,2, \ldots, K-1$, the $t$ th indices of the channel symbol index vectors at states belonging to the same subgroup of any level $t$ group must be the same, and they must be different from the $t$ th indices of the channel symbol index vectors at states belonging to any other subgroup of that group.

(2b) For $t=1,2, \ldots, K-2$, the $(t+1)$ st, $(t+2)$ nd,.. , $(K-1)$ st indices of the channel symbol index vectors at states belonging to the same level $t$ group must be the same.

\section{DESIGN FOR CODING ADVANTAGE}

In general, finding the best way to assign channel symbol indices to antennas and states is not a simple task. However, in the $N=N_{\min }$ case, we can find an efficient method to maximize the coding gain, so from now on it is assumed that the encoder has $N_{\min }$ states. 
The channel symbol difference matrix corresponding to the first $K$ long segment of the error paths of the first decoding error event is the matrix $D_{1}$. It is square and upper triangular, so its determinant is the product of its diagonal elements. Let us define the $K$ by $K$ matrix $\mathbf{A}_{1}$ as $\mathbf{A}_{1}=\mathbf{D}_{1} \mathbf{D}_{1}{ }^{H}$. Then the determinant of $\mathbf{A}_{1}$ is

$$
\operatorname{det}\left(\mathbf{A}_{\mathbf{1}}\right)=\prod_{k=0}^{K-1}\left|c\left(i^{k}\left(S_{k}, b_{k}\right)\right)-c\left(i^{k}\left(S_{k}^{\prime}, b_{k}^{\prime}\right)\right)\right|^{2}
$$

The matrix $\mathbf{D}$ can be decomposed as:

$$
\mathbf{D}=\left[\mathbf{D}_{1}, \mathbf{D}_{2}\right]
$$

where $\mathbf{D}_{\mathbf{2}}$ is a $K$ by $(T-K)$ matrix. The matrix $\mathbf{A}=$ $\mathrm{DD}^{H}$, whose minimum determinant is to be maximized, can be expressed as:

$$
\mathbf{A}=\mathbf{D}_{1} \mathbf{D}_{1}{ }^{H}+\mathbf{D}_{2} \mathbf{D}_{2}{ }^{H}=\mathbf{A}_{1}+\mathbf{A}_{2},
$$

where $\mathbf{A}_{\mathbf{2}}=\mathbf{D}_{\mathbf{2}} \mathbf{D}_{\mathbf{2}}{ }^{H}$. By construction, both $\mathbf{A}_{\mathbf{1}}$ and $\mathbf{A}_{\mathbf{2}}$ are Hermitian and nonnegative definite, so the eigenvalues of $\mathbf{A}$ are lower bounded by the eigenvalues of $\mathbf{A}_{\mathbf{1}}$. Therefore, the determinant of $\mathbf{A}$ satisfies the inequality

$$
\operatorname{det}(\mathbf{A}) \geq \operatorname{det}\left(\mathbf{A}_{\mathbf{1}}\right) \text {. }
$$

We can fix an arbitrary correct path and pick an arbitrary error path that is longer than $K$ state transitions. Both this error path and the error path corresponding to the $K$ long error event that starts from the same $S_{0}$ starting state and the same $b_{0}^{\prime}$ starting branch go through states that belong to the same subgroups of the same groups, resulting in $D_{1}$ matrices with the same diagonal elements. Therefore, for any error event that is longer than $K$ state transitions, it is possible to find a $K$ long error event with the same $\operatorname{det}\left(\mathbf{A}_{\mathbf{1}}\right)$ value. As a consequence of this observation and (3), $\gamma_{\min }$, the minimum determinant of the code can be expressed as:

$$
\gamma_{\min }=\min _{\substack{\left\{S_{l}, b_{l}\right\},\left\{S_{l}^{\prime}, b_{l}^{\prime}\right\} \\ l=0,1, \ldots, K-1}} \prod_{k=0}^{K-1}\left|c\left(i^{k}\left(S_{k}, b_{k}\right)\right)-c\left(i^{k}\left(S_{k}^{\prime}, b_{k}^{\prime}\right)\right)\right|^{2}
$$

The minimum is taken over all possible $K$ long correct and incorrect path segments.

The $\left\{S_{k}\right\}$ and $\left\{S_{k}^{\prime}\right\}$ state transition sequences can also be described by making use of the group/subgroup structure of the trellis. The results of Section III allow us to map the first $K$ long segment of the correct and erroneous paths of the first decoding error event onto different groups and subgroups of states. Toward this end, we introduce a channel symbol index based notation that does not explicitly depend on the state transition sequence.

Let $i_{l}^{0}, i_{l}^{0} \in\{0,1, \ldots, B-1\}$, be the 0 th indices of the channel symbol index vectors at the same state corresponding to source symbol $l(l \in\{0,1, \ldots, B-1\})$. For simplicity, it is assumed that the 0th indices of the channel symbol index vectors at different states corresponding to the same source symbol values are the same. Moreover, let $i_{l}^{k}$, $k=1,2, \ldots, K-1, i_{l}^{k} \in\{0,1, \ldots, B-1\}$, denote the $k$ th indices of the channel symbol index vectors at the states belonging to the $l$ th subgroup of the same level $k$ group $(l \in\{0,1, \ldots, B-1\})$. According to design rules (1a) and (2a), the relation $i_{l}^{k} \neq i_{m}^{k}$ must hold for any $l \neq m$. Therefore, the $B$-tuple $\left(i_{0}^{k}, i_{1}^{k}, \ldots, i_{B-1}^{k}\right), k=0,1, \ldots, K-1$, is an (arbitrary) permutation of the numbers $0,1, \ldots, B-1$.

Using Property 5 from Section III, we can make the following substitutions:

$$
i^{k}\left(S_{k}, b_{k}\right)=i_{l}^{k} \quad \text { and } \quad i^{k}\left(S_{k}^{\prime}, b_{k}^{\prime}\right)=i_{m}^{k},
$$

and the expression for the minimum determinant becomes:

$$
\gamma_{\text {min }}=\min _{\substack{l, m \in\{0,1, \ldots, B-1\} \\ l<m}} \prod_{k=0}^{K-1}\left|c\left(i_{l}^{k}\right)-c\left(i_{m}^{k}\right)\right|^{2} .
$$

The $l<m$ condition can be used since the squared distance function is symmetric in its arguments. The goal is to maximize the minimum determinant. Therefore, if $\Omega_{B}$ denotes the set of all permutations of the numbers $0,1, \ldots, B-1$, and $\sigma^{k} \in \Omega_{B}(k=0,1, \ldots, K-1)$ stands for a particular permutation $\left(i_{0}^{k}, i_{1}^{k}, \ldots, i_{B-1}^{k}\right)$, then $\gamma_{\text {min }}^{*}$, the optimal minimum determinant, can be expressed as:

$$
\gamma_{\min }^{*}=\max _{\sigma^{0}, \sigma^{1}, \ldots, \sigma^{K-1}} \gamma_{\min }
$$

Because the numbers $0,1, \ldots, B-1$ can be arranged in $B$ ! different ways, the size of the search space is $(B !)^{K}$. This means that exhaustive search may be impractical in certain cases. To get around this complexity growth, we propose a suboptimal approach that offers a practical solution. The basic idea of the method is to restrict the search space in such a way that the resulting complexity is not prohibitive.

The parametric permutation function is a function that generates a subset of all possible permutations of the numbers $0,1, \ldots, B-1$. Different parameters produce different permutations, so the problem will be reduced to a search for the best parameter.

In the sequel, we will use the notation $i_{2}=\psi_{B}\left(n, i_{1}\right)$ for one possible realization of the parametric permutation function. The value $n$ is the parameter to be optimized ( $n \in\{1,2, \ldots, B-1\}$ ), and $i_{1}$ and $i_{2}$ are the input and output indices, respectively $\left(i_{1}, i_{2} \in\{0,1, \ldots, B-1\}\right)$. Note that the obvious $\psi_{B}\left(n, i_{1}\right)=\left(n i_{1}\right) \bmod B$, for odd $n$, is not a good choice because it does not "shuffle" the indices well enough.

The output index is generated according to the following description. The input index $i_{1}$ and the parameter $n$ are treated as binary vector representations of two field elements in $\mathrm{GF}(B)=\mathrm{GF}\left(2^{b}\right)$. These field elements are multiplied together according to field arithmetic, and the output index $i_{2}$ will be the binary vector representation of the product.

Replacing the permutation operation by the parametric permutation function, the optimization problem reduces to:

$$
\gamma_{\min }^{*}=\max _{\substack{n_{0}, n_{1}, \ldots, n_{K-1} \\ \in\{1,2, \ldots, B-1\}}} \gamma_{\min }
$$


TABLE I

THE GENERATED PERMUTÁTIONS

\begin{tabular}{|c||c|c|c|}
\hline$i_{1}$ & $n=1$ & $n=2$ & $n=3$ \\
\hline \hline 0 & 0 & 0 & 0 \\
1 & 1 & 2 & 3 \\
2 & 2 & 3 & 1 \\
3 & 3 & 1 & 2 \\
\hline
\end{tabular}

where $\gamma_{\min }$ is:

$$
\gamma_{\min }=\min _{\substack{l, m \in\{0,1, \ldots, B-1\} \\ i<m}} \prod_{k=0}^{K-1}\left|c\left(\psi_{B}\left(n_{k}, l\right)\right)-c\left(\psi_{B}\left(n_{k}, m\right)\right)\right|^{2} .
$$

To find the maximum of $\gamma_{\text {min }}$, we only have to search over $(B-1)^{K}$ possibilities. Once the $n_{0}^{*}, n_{1}^{*}, \ldots, n_{K-1}^{*}$ parameter values that maximize the minimum determinant have been calculated, the channel symbol indices can be determined as $i_{l}^{k}=\psi_{B}\left(n_{k}^{*}, l\right)$, for $k=0,1, \ldots, K-1$ and $l=0,1, \ldots, B-1$.

The delay diversity scheme [5] is a special case of our design method. For $B=2$, the two methods are equivalent. For $B>2$, the delay diversity scheme corresponds to $i_{l}^{k}=$ $l$. In [5], it was shown that, if $\Delta$ denotes the minimum Euclidean distance of the chosen constellation, then the minimum determinant of the resulting delay diversity ST code will be $\gamma_{\min }^{D}=\Delta^{2 K}$.

\section{A CODE DESIGN EXAMPLE}

In this section, we provide a ST code construction example for 3.transmit antennas and QPSK modulation.

The parametric permutation function is calculated using the field arithmetic in GF(4). The field is built up using $\alpha$, a root of the primitive polynomial $p(x)=x^{2}+x+1$. In this case, the function $\psi_{4}(\cdot, \cdot)$ will generate the permutations given in Table I. The table entries are the function values for different input index and parameter values.

The minimum determinant of the code can be expressed as:

$$
\gamma_{\min }=\min _{\substack{l, m \\ \in\{, 1,2,3\} \\ i<m}} 4^{3} \prod_{k=0}^{2} \sin ^{2}\left(\left(\psi_{4}\left(n_{k}, l\right)-\psi_{4}\left(n_{k}, m\right)\right) \frac{\pi}{4}\right) .
$$

If the optimization procedure described in Section IV is used, then the result will be $n_{0}^{*}=1, n_{1}^{*}=2$ and $n_{2}^{*}=3$ with $\gamma_{\min }^{*}=16$. Note that this maximum is not unique: several other sets of $\left\{n_{k}^{*}\right\}$ values exist. This is not surprising because of the symmetry of the QPSK constellation and the commutativity of multiplication. The obtained permutations are: $\left(i_{0}^{0}, i_{1}^{0}, i_{2}^{0}, i_{3}^{0}\right)=(0,1,2,3),\left(i_{0}^{1}, i_{1}^{1}, i_{2}^{1}, i_{3}^{1}\right)=$ $(0,2,3,1)$, and $\left(i_{0}^{2}, i_{1}^{2}, i_{2}^{2}, i_{3}^{2}\right)=(0,3,1,2)$. These permutations generate the ST code depicted in Figure 1. The minimum determinant of the corresponding delay diversity ST code is $\gamma_{\min }^{D}=\Delta^{6}=8$.

\section{Simulation Results}

To illustrate the performance of the codes designed using the above described method, we show some simula-

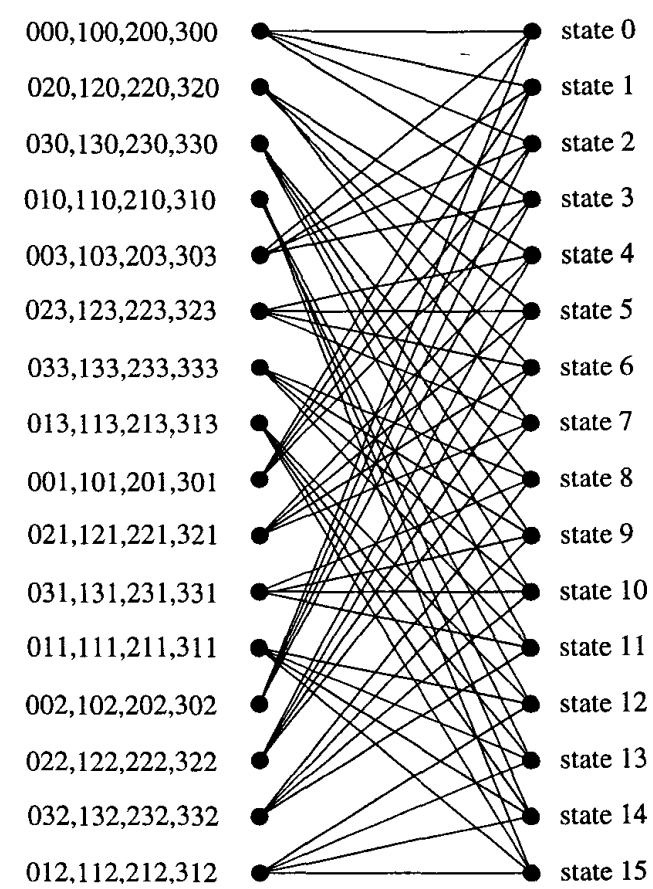

Fig. 1. Example ST code for 3 antennas and QPSK constellation

tion results. We compare our method with the delay diversity scheme of [5] since, to our knowledge, this is the only method that can be used to construct ST codes for any number of antennas and any constellation. The simulated communication system had one receive antenna. The source symbols were transmitted in frames of length 130 , and the Viterbi algorithm with decoding depth of 20 state transitions was used to decode the received signals. For each frame, the path gains between the transmit antennas and the receive antenna were modeled as independent, complex, zero mean, circularly symmetric Gaussian random variables with unit variance.

Since the frame error probability depends on the length of the frame and it does not seem very informative, we present probability of bit error curves as functions of the average signal to noise ratio (SNR) per source symbol at the receive antenna. In the sequel, the expression coding gain will refer to the difference (in $\mathrm{dB}$ ) of transmit energies to achieve the same probability of bit error value.

Figure 2 depicts the performance of the example ST code for 3 antennas and QPSK modulation. Approximately 0.4$0.5 \mathrm{~dB}$ coding gain is observed over the delay diversity scheme.

Figure 3 shows the bit error rate curves of a 3 transmit antenna system with 8PSK modulation. The minimum determinant of the code is $\gamma_{\min }^{*}=0.6863$. The minimum determinant of the delay diversity scheme with the same design parameters is $\gamma_{\min }^{D}=0.2010$. The simulation also shows that the performance improvement is more pronounced; at higher SNR, more than $1 \mathrm{~dB}$ coding gain can be achieved. 


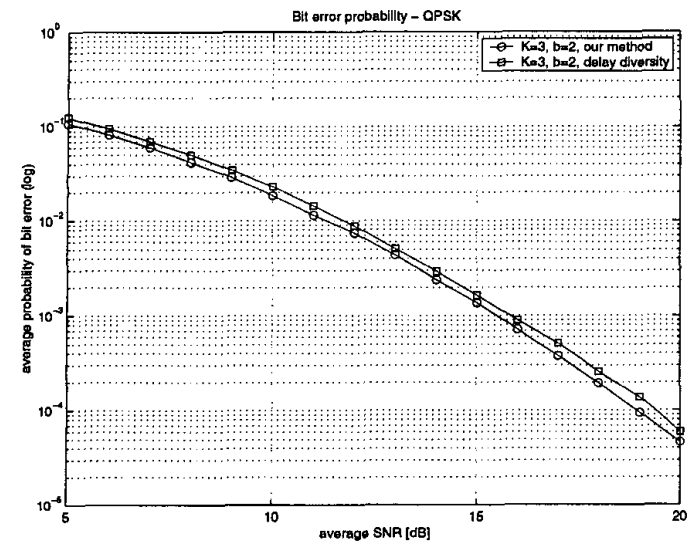

Fig. 2. 3 transmit antennas with QPSK

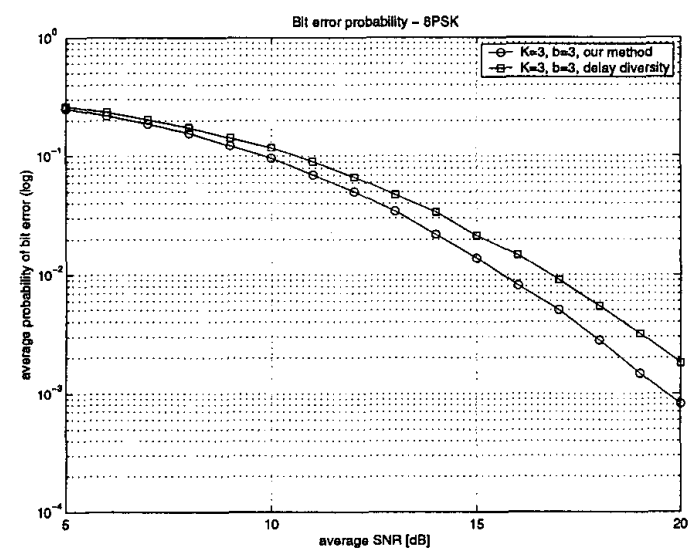

Fig. 3. 3 transmit antennas with 8PSK

The performance of the ST code for 3 antennas and 16PSK constellation can be observed in Figure 4. The minimum determinant of this code is $\gamma_{m i n}^{*}=0.110105$, while the delay diversity construction gives $\gamma_{\min }^{D}=0.003529$. Our ST code yields $2-2.5 \mathrm{~dB}$ coding gain compared to the delay diversity scheme.

Figure 5 depicts the bit error rate curves for the 4 antenna ST code using 4ASK modulation. To normalize the constellation, the distance between the neighboring signal points was set to $\Delta=\sqrt{4 / 5}$. The minimum determinant of the code is $\gamma_{\min }^{*}=1.6384$, and the delay diversity method yields $\gamma_{\min }^{D}=0.4096$. The figure shows approximately 2 $\mathrm{dB}$ coding gain over the delay diversity construction.

\section{CONCLUSION}

Exploiting the special structure of the code difference matrix, we developed a systematic method to design ST codes for both diversity advantage and coding advantage for an arbitrary number of transmit antennas and any memoryless constellation. Based on the theoretical coding advantage values and the simulation results, we can draw the following conclusion. If the maximum distance of the chosen constellation is much larger than the min-

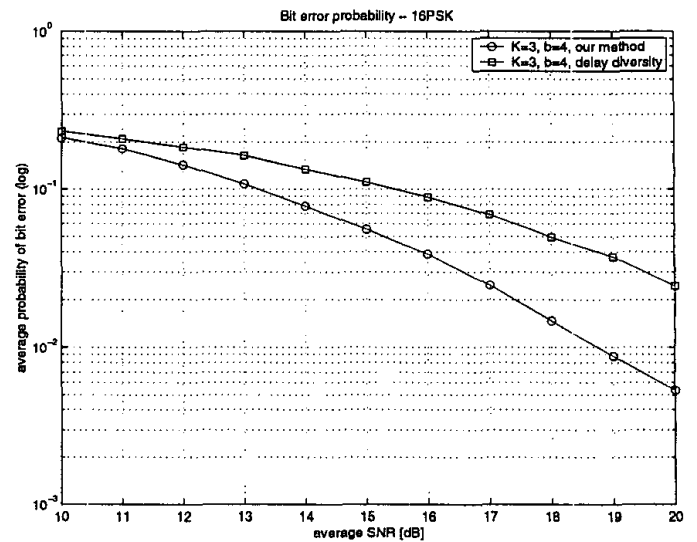

Fig. 4. 3 transmit antennas with 16PSK

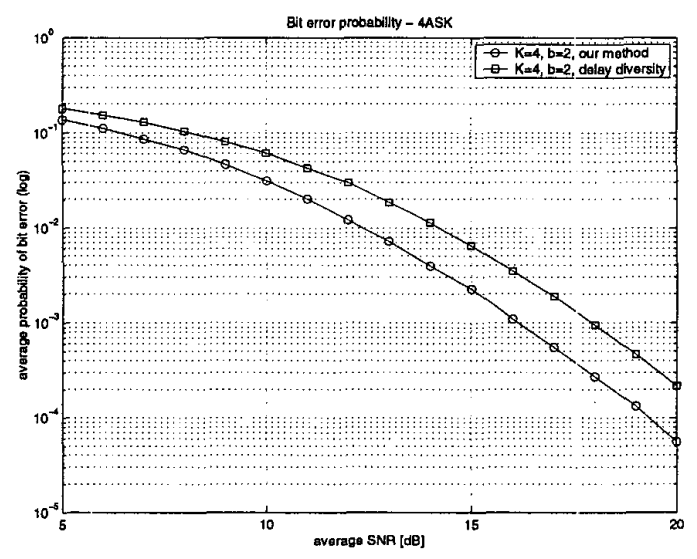

Fig. 5. 4 transmit antennas with 4ASTK

imum distance, our design method can exploit the additional degrees of freedom effectively, producing ST codes that perform much better than the delay diversity scheme.

\section{REFERENCES}

[1] I. Telatar, Capacity of Multi-Antenna Gaussian Channels, Technical Report, AT\&T Bell Labs, 1995.

[2] G. Foschini, Layered Space-Time Architecture for Wireless Communication in a Fading Environment When Using Multi-Element Antennas, Bell Labs Technical Journal, Vol. 1, No. 2, pp. 41-59, 1996.

[3] J. Guey, M. Fitz, M. Bell and W. Kuo, Signal Design for Transmitter Diversity Wireless Communication Systems over Rayleigh Fading Channels, IEEE Vehicular Technology Conference, pp. $136-140,1996$.

[4] V. Tarokh, N. Seshadri, A. Calderbank, Space-Time Codes for High Data Rate Wireless Communication : Performance Criterion and Code Construction, IEEE Transactions on Information Theory, Vol. 44, No. 2, pp. 744-765, March 1998.

[5] J. Grimm and M. Fitz and J. Krogmeier, Further Results on Space-Time Coding for Rayleigh Fading, Proceedings of the 36th Allerton Conference on Communications, Control and Computing, pp. 391-400, 1998.

[6] A. R. Hammons and H. El Gamal, On the Theory of Space-Time Codes for PSK Modulation, IEEE Transactions on Information Theory, Vol. 46, No. 2, pp. 524-542, March 2000.

[7] Z. Safar and K.J.R. Liu, Design of Space-Time Trellis Codes for Full Spatial Diversity, Proceedings of ICASSP, May 2001. 